\title{
LINEAR FINITE ELEMENTS MAY BE ONLY FIRST-ORDER POINTWISE ACCURATE ON ANISOTROPIC TRIANGULATIONS
}

\author{
NATALIA KOPTEVA
}

\begin{abstract}
We give a counterexample of an anisotropic triangulation on which the exact solution has a second-order error of linear interpolation, while the computed solution obtained using linear finite elements is only first-order pointwise accurate. Our example is given in the context of a singularly perturbed reaction-diffusion equation, whose exact solution exhibits a sharp boundary layer. Furthermore, we give a theoretical justification of the observed numerical phenomena using a finite-difference representation of the considered finite element methods. Both standard and lumped-mass cases are addressed.
\end{abstract}

\section{INTRODUCTION}

It appears that there is a perception in the finite-element community that the computed-solution error in the maximum norm is closely related to the corresponding interpolation error. While an almost best approximation property of finiteelement solutions in the maximum norm has been rigourously proved (with a logarithmic factor in the case of linear elements) for some equations on quasi-uniform meshes [12,13, there is no such result for strongly-anisotropic triangulations. Nevertheless, this perception is frequently considered a reasonable heuristic conjecture to be used in the anisotropic mesh adaptation [4, 6, 8,

In this note we give a counterexample of an anisotropic triangulation on which

- the exact solution is in $C^{\infty}(\bar{\Omega})$ and has a second-order pointwise error of linear interpolation $O\left(N^{-2}\right)$,

- the computed solution obtained using linear finite elements is only firstorder pointwise accurate, i.e., the pointwise error is as large as $O\left(N^{-1}\right)$.

Here the maximum side length of mesh elements is $O\left(N^{-1}\right)$ and the global number of mesh nodes does not exceed $O\left(N^{2}\right)$.

Our example is given in the context of singularly perturbed differential equations. Their solutions exhibit sharp boundary and interior layers, so locally anisotropic meshes (fine and anisotropic in layer regions and standard outside) are frequently employed in their numerical solution and, furthermore, have been shown to yield reliable numerical approximations in an efficient way (see, e.g., [5, 9, 14 and references in 10$]$ ).

Received by the editor September 14, 2012 and, in revised form, January 22, 2013.

2010 Mathematics Subject Classification. Primary 65N15, 65N30, 65N50; Secondary 65N06.

Key words and phrases. Anisotropic triangulation, linear finite elements, maximum norm, singular perturbation, Bakhvalov mesh, Shishkin mesh. 

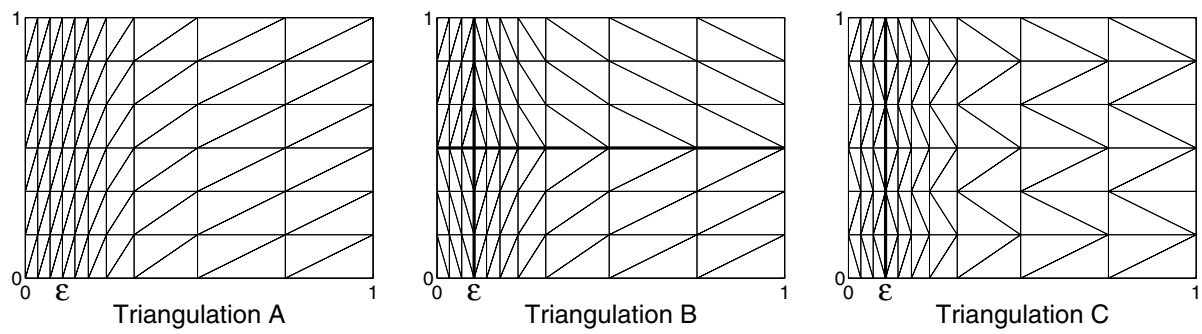

FiguRE 1. Triangulations $\mathrm{A}, \mathrm{B}$ and $\mathrm{C}$ are obtained from the same layer-adapted tensor-product mesh by drawing diagonals in a different manner. The bold lines indicate a change in the type of triangulation.

Example. Consider an exact solution $u(x, y)=e^{-x / \varepsilon}$ of the singularly perturbed reaction-diffusion problem

$$
-\varepsilon^{2} \Delta u+u=0 \quad \text { for }(x, y) \in \Omega, \quad u=g \quad \text { for }(x, y) \in \partial \Omega,
$$

where $\varepsilon$ is a small positive parameter, $\Delta=\partial^{2} / \partial x^{2}+\partial^{2} / \partial y^{2}$ is the Laplace operator, and the boundary data $g$ matches the chosen exact solution. The domain $\Omega$ is a bounded polygonal domain. In particular we consider the cases of $\Omega=(0,1)^{2}$ and $\Omega=(0,2 \varepsilon) \times(0,1)$, as well as a more general case of $\Omega \supset \stackrel{\Omega}{\Omega}:=(0,2 \varepsilon) \times(-H, H)$, where $H=O\left(N^{-1}\right)$.

The paper is organized as follows. In $₫ 2$, problem (1.1) is solved numerically using both standard and lumped-mass linear finite elements. Triangulations of types A, B and $\mathrm{C}$ are considered that are obtained from standard layer-adapted tensor-product meshes by drawing diagonals as in Figure 1, In $₫ 3$, we give a theoretical justification of the observed numerical phenomena using a finite-difference representation of the considered finite element methods.

Notation. We let $C$ denote a generic positive constant that may take different values in different formulas, but is always independent of the mesh and $\varepsilon$. A subscripted $C$ (e.g., $C_{1}$ ) denotes a positive constant that is independent of $N$ and $\varepsilon$ and takes a fixed value. Notation $v=O(w)$ will be used for $C^{-1} w \leq v \leq C w$, while $v=\mathcal{O}(w)$ will denote $|v| \leq C w$, with some constant $C$.

\section{Numerical RESUlts}

Consider a tensor product of a layer-adapted mesh $\left\{x_{i}\right\}_{i=0}^{N}$ in the $x$-direction and the uniform mesh $\left\{\frac{j}{M}\right\}_{j=0}^{M}$ in the $y$-direction, where $M=O(N)$. Whenever $\Omega=(0,1)^{2}$, the mesh $\left\{x_{i}\right\}$ will be a version of the Bakhvalov mesh [2] or the Shishkin mesh [14] described below. Whenever $\Omega=(0,2 \varepsilon) \times(0,1)$, the mesh $\left\{x_{i}\right\}$ will be uniform.

Bakhvalov mesh 22. For some $\gamma \in(0,1)$, set $\sigma:=2 \varepsilon\left(\gamma^{-1}|\ln \varepsilon|+1\right)$ and assume that $\varepsilon$ is sufficiently small for $\sigma \in(0,1)$. Now define the mesh $\left\{x_{i}\right\}_{i=0}^{N / 2}$ on $[0, \sigma]$ by

$$
x_{i}:=x\left([1+\gamma-\varepsilon] \frac{2 i}{N}\right), \quad x(t):=\left\{\begin{array}{cl}
2 \varepsilon \gamma^{-1} t, & t \in[0, \gamma], \\
2 \varepsilon\left[1-\gamma^{-1} \ln (1+\gamma-t)\right], & t \in[\gamma, 1+\gamma-\varepsilon] .
\end{array}\right.
$$

The remaining part of the mesh $\left\{x_{i}\right\}_{i=N / 2}^{N}$ on $[\sigma, 1]$ is uniform. In fact, one can easily see that on $[0,2 \varepsilon]$ this mesh is also uniform, with $x_{i}-x_{i-1}=O\left(\varepsilon N^{-1}\right)$. 
TABLE 1. Bakhvalov tensor-product mesh, $\gamma=0.8, M=\frac{1}{4} N$ : maximum nodal errors and computational rates $r$ in $\left(N^{-1}\right)^{r}$.

\begin{tabular}{|c|c|c|c|c|c|c|c|}
\hline & $N$ & $\begin{array}{l}\text { Triangul } \\
\varepsilon=2^{-8}\end{array}$ & $\begin{array}{l}\operatorname{tion} \mathrm{A} \\
\varepsilon=2^{-16}\end{array}$ & $\varepsilon=2^{-24}$ & $\begin{array}{l}\text { Triangul } \\
\varepsilon=2^{-8}\end{array}$ & $\begin{array}{l}\text { tion B } \\
\varepsilon=2^{-16}\end{array}$ & $\varepsilon=2^{-24}$ \\
\hline \multirow{7}{*}{ 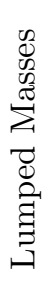 } & 32 & $1.065 \mathrm{e}-3$ & $1.070 \mathrm{e}-3$ & $1.070 \mathrm{e}-3$ & $1.543 \mathrm{e}-2$ & $1.545 \mathrm{e}-2$ & $1.545 \mathrm{e}-2$ \\
\hline & & 1.99 & 1.99 & 1.99 & 1.07 & 1.07 & 1.07 \\
\hline & 64 & $2.685 \mathrm{e}-4$ & $2.697 \mathrm{e}-4$ & $2.697 \mathrm{e}-4$ & $7.328 \mathrm{e}-3$ & $7.353 \mathrm{e}-3$ & $7.353 \mathrm{e}-3$ \\
\hline & & 2.00 & 2.00 & 2.00 & 0.98 & 0.97 & 0.97 \\
\hline & 128 & $6.726 \mathrm{e}-5$ & $6.756 \mathrm{e}-5$ & $6.756 \mathrm{e}-5$ & $3.713 \mathrm{e}-3$ & $3.757 \mathrm{e}-3$ & $3.757 \mathrm{e}-3$ \\
\hline & & 2.00 & 2.00 & 2.00 & 1.03 & 0.99 & 0.99 \\
\hline & 256 & $1.684 \mathrm{e}-5$ & $1.691 \mathrm{e}-5$ & $1.691 \mathrm{e}-5$ & $1.818 \mathrm{e}-3$ & $1.897 \mathrm{e}-3$ & $1.897 \mathrm{e}-3$ \\
\hline \multirow{7}{*}{ 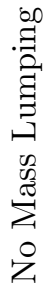 } & \multirow[t]{2}{*}{32} & $1.422 \mathrm{e}-3$ & $1.430 \mathrm{e}-3$ & $1.430 \mathrm{e}-3$ & $1.718 \mathrm{e}-2$ & $1.723 \mathrm{e}-2$ & $1.723 \mathrm{e}-2$ \\
\hline & & 2.01 & 2.01 & 2.01 & 0.97 & 0.97 & 0.97 \\
\hline & \multirow[t]{2}{*}{64} & $3.526 \mathrm{e}-4$ & $3.554 \mathrm{e}-4$ & $3.554 \mathrm{e}-4$ & $8.754 \mathrm{e}-3$ & $8.811 \mathrm{e}-3$ & $8.811 \mathrm{e}-3$ \\
\hline & & 2.02 & 2.00 & 2.00 & 0.98 & 0.96 & 0.96 \\
\hline & \multirow[t]{2}{*}{128} & $8.710 \mathrm{e}-5$ & $8.873 \mathrm{e}-5$ & $8.873 \mathrm{e}-5$ & $4.443 \mathrm{e}-3$ & $4.527 \mathrm{e}-3$ & $4.527 \mathrm{e}-3$ \\
\hline & & 2.05 & 2.00 & 2.00 & 1.04 & 0.98 & 0.98 \\
\hline & 256 & $2.097 \mathrm{e}-5$ & $2.217 \mathrm{e}-5$ & $2.217 \mathrm{e}-5$ & $2.156 \mathrm{e}-3$ & $2.292 \mathrm{e}-3$ & $2.292 \mathrm{e}-3$ \\
\hline
\end{tabular}

Shishkin mesh [14]. For some $\gamma \in(0,1)$, set $\sigma=2 \gamma^{-1} \varepsilon \ln \frac{N}{2}$. Now construct a piecewise uniform mesh by dividing the intervals $[0, \sigma]$ and $[\sigma, 1]$ into $N_{1}$ and $N-N_{1}$ equal subintervals for some $N_{1}=O(N)$.

Remark 2.1 (Interpolation error). A calculation shows that the linear interpolation error of our exact solution $u=e^{-x / \varepsilon}$ on any of the Triangulations A, B or C is $O\left(\left[N^{-1} \ln ^{p} \frac{N}{2}\right]^{2}\right)$ in the maximum norm, where $p=0$ if the Bakhvalov mesh is used or the uniform mesh in the domain $\Omega=(0,2 \varepsilon) \times(0,1)$, and $p=1$ if the Shishkin mesh is used. Interestingly, one gets a similar second-order bound (with a logarithmic factor in the case of the Shishkin mesh) for the error of the standard five-point difference scheme applied to problem (1.1) on the corresponding tensorproduct mesh (see, e.g., [5, 9]).

Tables 1-3 give the maximum nodal errors (odd rows) and the computational convergence rates $r$ in $\left(N^{-1} \ln ^{p} \frac{N}{2}\right)^{r}$ (even rows) for Triangulations A and B obtained from the three tensor-product meshes. For the considered values of $\varepsilon$, these meshes are highly anisotropic; for example, in the case of the Bakhvalov mesh (see Table 1), the mesh aspect ratio changes between 2 away from the layer and $(2.25 \varepsilon)^{-1}$ in the layer region. The numerical results for the standard finite elements are quite similar to the case of mass lumping, so we consider both cases only in Table 1.

We observe that whenever Triangulation $\mathrm{A}$ is used, one gets a second-order accuracy, with a logarithmic factor in the case of the Shishkin mesh (similar to the accuracy of the five-point finite-difference scheme, which, in fact, is identical with the lumped-mass finite elements on Triangulation A). However, when one switches to Triangulation B, linear finite elements become only (almost) first-order pointwise accurate in contrast to the (almost) second-order accuracy of the interpolation error. Triangulation $\mathrm{C}$ also yields only (almost) first-order convergence (see also Remark 3.6] and Figure 31). 
TABLE 2. Shishkin mesh, $\gamma=0.9, M=\frac{1}{4} N, N_{1}=\frac{3}{4} N$ : maximum nodal errors and computational rates $r$ in $\left(N^{-1} \ln \frac{N}{2}\right)^{r}$.

\begin{tabular}{|c|c|c|c|c|c|c|c|}
\hline & $N$ & $\begin{array}{l}\text { Triangul } \\
\varepsilon=2^{-8}\end{array}$ & $\begin{array}{l}\text { tion A } \\
\varepsilon=2^{-16}\end{array}$ & $\varepsilon=2^{-24}$ & $\begin{array}{l}\text { Triangul } \\
\varepsilon=2^{-8}\end{array}$ & $\begin{array}{l}\text { tion B } \\
\varepsilon=2^{-16}\end{array}$ & $\varepsilon=2^{-24}$ \\
\hline \multirow{7}{*}{ 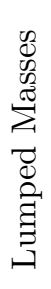 } & \multirow[t]{2}{*}{32} & $1.850 \mathrm{e}-3$ & $2.109 \mathrm{e}-3$ & $2.109 \mathrm{e}-3$ & $1.477 \mathrm{e}-2$ & $1.478 \mathrm{e}-2$ & $1.478 \mathrm{e}-2$ \\
\hline & & 3.30 & 3.28 & 3.28 & 1.22 & 1.22 & 1.22 \\
\hline & \multirow[t]{2}{*}{64} & $3.930 \mathrm{e}-4$ & $4.516 \mathrm{e}-4$ & $4.521 \mathrm{e}-4$ & $8.317 \mathrm{e}-3$ & $8.340 \mathrm{e}-3$ & $8.340 \mathrm{e}-3$ \\
\hline & & 2.00 & 2.27 & 2.27 & 0.98 & 0.96 & 0.96 \\
\hline & \multirow[t]{2}{*}{128} & $1.418 \mathrm{e}-4$ & $1.418 \mathrm{e}-4$ & $1.418 \mathrm{e}-4$ & $5.048 \mathrm{e}-3$ & $5.103 \mathrm{e}-3$ & $5.103 \mathrm{e}-3$ \\
\hline & & 2.00 & 2.00 & 2.00 & 1.02 & 0.97 & 0.97 \\
\hline & 256 & $4.832 \mathrm{e}-5$ & $4.832 \mathrm{e}-5$ & $4.832 \mathrm{e}-5$ & $2.908 \mathrm{e}-3$ & $3.027 \mathrm{e}-3$ & $3.027 \mathrm{e}-3$ \\
\hline
\end{tabular}

TABLE 3. Uniform mesh in $(0,2 \varepsilon) \times(0,1), M=\frac{1}{4} N$ : maximum nodal errors and computational rates $r$ in $\left(N^{-1}\right)^{r}$.

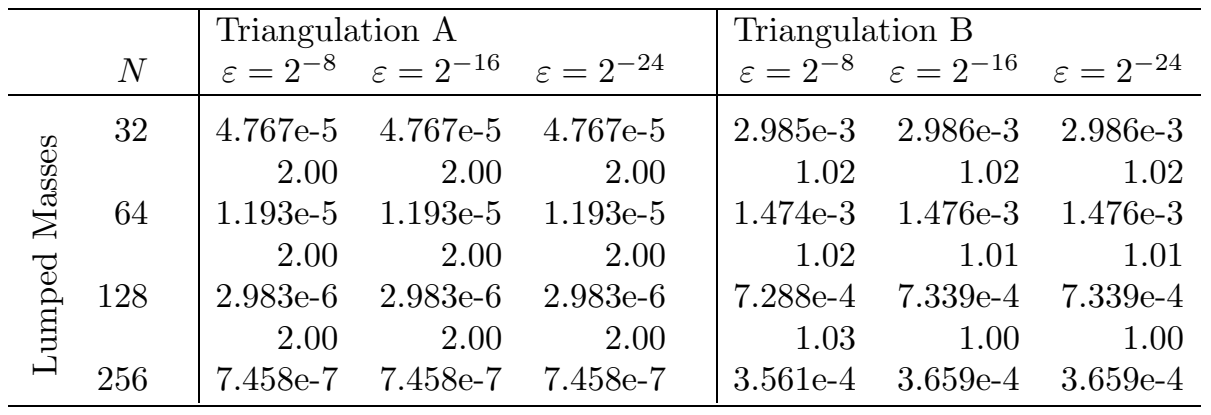

In summary, we conclude that

- when Triangulations B and C are used, linear finite elements are only firstorder pointwise accurate;

- the order of convergence dramatically deteriorates from 2 to 1 as one switches from Triangulation A to Triangulation B or C.

Remark 2.2 (Comparison with the $L_{2}$ projection error). The $L_{2}$ projection errors are less understood on general anisotropic meshes. But one can easily estimate the pointwise error of this projection for the triangulations addressed in Table 3, i.e., obtained from the uniform tensor-product mesh in the domain $(0,2 \varepsilon) \times(0,1)$. Indeed, the stretching transformation $\hat{x}:=x / \varepsilon$ maps any of Triangulations $\mathrm{A}, \mathrm{B}$ or $\mathrm{C}$ of the domain $(0,2 \varepsilon) \times(0,1)$ into a quasi-uniform triangulation in the domain $(0,2) \times(0,1)$. Denoting the $L_{2}$ projection operators on the original and stretched triangulations by $P$ and $\hat{P}$, respectively, one gets $P u(x, y)=\hat{P} u(\varepsilon \hat{x}, y)$. As on the quasi-uniform stretched triangulation $\hat{P} u(\varepsilon \hat{x}, y)-u(\varepsilon \hat{x}, y)=\mathcal{O}\left(N^{-2}\right)$, the $L_{2}$ projection error bound $P u(x, y)-u(x, y)=\mathcal{O}\left(N^{-2}\right)$ follows. So in this case, the exact solution has a second-order pointwise error of the $L_{2}$ projection, while, as Table 3 demonstrates, the pointwise error of the computed solution obtained using linear finite elements may be only first-order pointwise accurate. 
TABLE 4. Normalized $L_{2}$ norm errors $\varepsilon^{-1 / 2}\left\|U-u^{I}\right\|_{L_{2}(\Omega)}$ and computational rates $r$ in $\left(N^{-1}\right)^{r}$ : Bakhvalov tensor-product mesh, $\gamma=0.8, M=\frac{1}{4} N$.

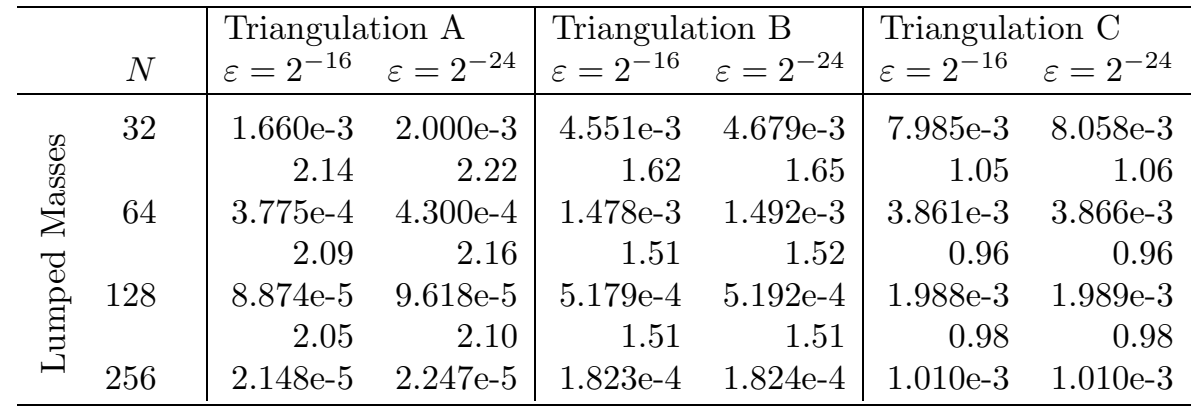

Errors in the $L_{2}$ norm. Although our main interest in this paper is in the pointwise accuracy of computed solutions, in Table 4 we also list the normalized $L_{2}$ norm errors $\varepsilon^{-1 / 2}\left\|U-u^{I}\right\|_{L_{2}(\Omega)}$ (odd rows) and the computational convergence rates $r$ in $\left(N^{-1}\right)^{r}$, where $u^{I}$ is the piecewise-linear interpolant of $u$, for the Bakhvalov mesh. The table suggests that $\left\|U-u^{I}\right\|_{L_{2}(\Omega)} \approx O\left(\sqrt{\varepsilon} N^{-q}\right)$, where $q$ is $2, \frac{3}{2}$ and 1 for Triangulations A, B and C, respectively. Thus, although Triangulations B and $\mathrm{C}$ yield very similar maximum nodal errors, the latter is superior when the errors are computed in the $L_{2}$ norm (see also Figure 3, and Remark 3.6 for a theoretical explanation). The numerical results for the standard finite elements are quite similar to the case of mass lumping, so are not presented here.

When we switch to the uniform tensor-product mesh in the domain $(0,2 \varepsilon) \times(0,1)$ (as used in Table 3 ) to obtain triangulations of type A, B and C, the numerical results again suggest that $\left\|U-u^{I}\right\|_{L_{2}(\Omega)} \approx O\left(\sqrt{\varepsilon} N^{-q}\right)$. Finally, for the Shishkin mesh (as used in Table 2), one computationally observes that $\left\|U-u^{I}\right\|_{L_{2}\left(\Omega_{\sigma}\right)} \approx$ $O\left(\sqrt{\varepsilon} N^{-q}\left[\ln \frac{N}{2}\right]^{\max \{1,2 q-2\}}\right)$, where $\Omega_{\sigma}:=(0, \sigma) \times(0,1)$ is essentially the boundarylayer region (while the $L_{2}\left(\Omega \backslash \Omega_{\sigma}\right)$ norm of the error is quite small $\varepsilon$-independently).

It should also be noted that these results are obtained for the exact solution $e^{-x / \varepsilon}$, which is negligible away from the boundary-layer region. In general, the errors in the $L_{2}$ norm may involve an additional component $\mathcal{O}\left(N^{-2}\right)$ induced by a nontrivial smooth part of the exact solution.

\section{TheORETICAL JUSTIFICATION}

To understand the numerical phenomena described in the previous section, we represent the considered finite elements as finite-difference schemes on the underlying rectangular tensor-product meshes.

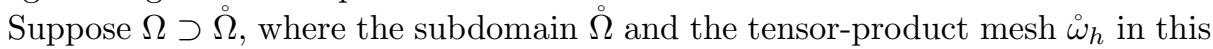
subdomain are defined by

$$
\stackrel{\Omega}{\Omega}:=(0,2 \varepsilon) \times(-H, H), \quad \stackrel{\circ}{\omega}_{h}:=\left\{x_{i}=h i\right\}_{i=0}^{2 N_{0}} \times\{-H, 0, H\}, \quad h=\frac{\varepsilon}{N_{0}} .
$$

The triangulation $\mathcal{T}^{\circ}$ in $\stackrel{\Omega}{\Omega}$ is obtained by drawing diagonals in each rectangle as shown on Figure 2 using the mesh transition point $(\varepsilon, 0)$. 

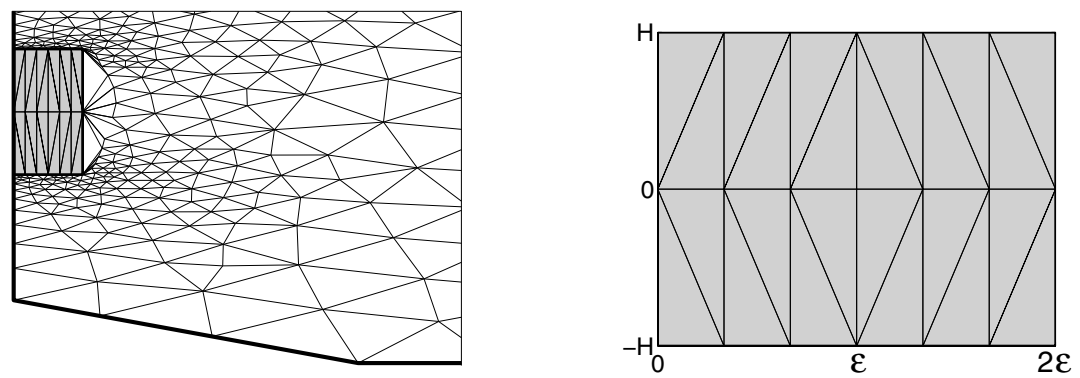

FigURE 2. Triangulation in $\Omega$; right-hand picture enlarges the subdomain $\Omega$.

Next, let $U$ be the piecewise-linear finite-element solution obtained on some triangulation $\mathcal{T} \supset \mathcal{T}^{\circ}$ in the global domain $\Omega$, for whose nodal values in $\Omega$ we use the notation

$$
U_{i}:=U\left(x_{i}, 0\right), \quad U_{i}^{ \pm}:=U\left(x_{i}, \pm H\right) .
$$

Now a calculation yields a finite-difference representation in the lumped-mass case:

$$
\mathcal{L}^{h} U\left(x_{i}, 0\right):=\frac{\varepsilon^{2}}{h^{2}}\left[-U_{i-1}+2 U_{i}-U_{i+1}\right]+\frac{\varepsilon^{2}}{H^{2}}\left[-U_{i}^{-}+2 U_{i}-U_{i}^{+}\right]+\gamma_{i} U_{i}=0
$$

for $i=1, \ldots, 2 N_{0}-1$, where

$$
\gamma_{i}=1 \quad \text { for } i \neq N_{0}, \quad \gamma_{N_{0}}=\frac{2}{3} .
$$

In the standard (non-lumped-mass) case, one gets a similar finite-difference representation for $i=1, \ldots, 2 N_{0}-1$ :

$$
\tilde{\mathcal{L}}^{h} U\left(x_{i}, 0\right):=\mathcal{L}^{h} U_{i}-\frac{1}{12} \sum_{\left(x^{\prime}, y^{\prime}\right) \in \mathcal{S}_{i}}\left[U_{i}-U\left(x^{\prime}, y^{\prime}\right)\right]=0,
$$

where $\mathcal{S}_{i}$ denotes the set of meshnodes that have a common edge with $\left(x_{i}, 0\right)$.

Remark 3.1. Note that if $\gamma_{i}$ in (3.2a) is replaced by 1 , one gets the standard fivepoint difference scheme for equation (1.1), for which, using the discrete maximum principle, one can easily show that if a tensor-product mesh of type $\stackrel{\circ}{\omega}_{h}$ is used in the entire domain $\Omega$, then the nodal error is $O\left(\frac{h^{2}}{\varepsilon^{2}}\right)$. Our method differs from this difference scheme at one point $\left(x_{N_{0}}, 0\right)$, where $\gamma_{i}=\frac{2}{3}$, i.e., at this point, compared to the second-order finite-difference method, we have a truncation error $O(1)$. As we show in Lemma 3.3 below, this results in the deterioration of pointwise accuracy of the computed solution to $O\left(\frac{h}{\varepsilon}\right)$.

Remark 3.2. A triangulation, similar to Triangulation B on Figure 1 (and also to

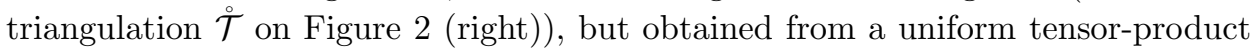
mesh, has been used to show that the logarithmic factor in the $L_{\infty}$-norm error estimate for linear finite elements applied to the Laplace equation is sharp [1, 3]. Our approach is, in fact, similar to that in [1] in that we represent a finite element method as a finite-difference scheme to get a lower bound for the error.

We shall consider the lumped-mass and non-lumped-mass cases separately. 


\subsection{Lumped-mass linear finite elements.}

Lemma 3.3 (Lumped-mass case). Let $u=e^{-x / \varepsilon}$ be the exact solution of prob-

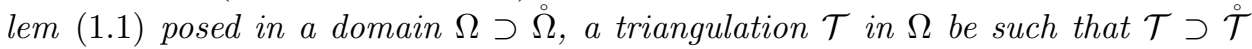
subject to the definitions (3.1), and let $U$ be the computed solution obtained using lumped-mass linear finite elements. For any positive constant $C_{2}$, there exist sufficiently small constants $C_{0}$ and $C_{1}$ such that if $N_{0}^{-1} \leq C_{1}$ and $\varepsilon \leq C_{2} H$, then

$$
\max _{\bar{\Omega}}|U-u| \geq C_{0} N_{0}^{-1} \text {. }
$$

Proof. (i) First, consider the auxiliary piecewise-linear computed solution $\stackrel{\circ}{U}$ obtained on the triangulation $\mathcal{T}^{\circ}$ in the subdomain $\stackrel{\Omega}{\Omega}$, subject to the boundary con-

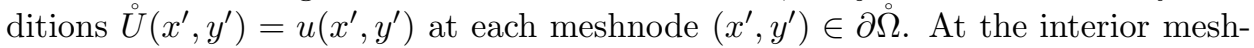
nodes, $\stackrel{\circ}{U}$ satisfies (3.2a) , i.e., $\left.\mathcal{L}^{h} \stackrel{\circ}{U}^{(} x_{i}, 0\right)=0$ for $i=1, \ldots, 2 N_{0}-1$. We shall now prove that, for a sufficiently small constant $C_{0}$, one has

$$
[\stackrel{\circ}{U}-u]\left(x_{N_{0}}, 0\right) \geq 2 C_{0} N_{0}^{-1} .
$$

Let $e:=\stackrel{\circ}{U}-u$ and $e_{i}:=e\left(x_{i}, 0\right)$. Recall that $e\left(x_{i}, \pm H\right)=0$, so $\mathcal{L}^{h} e\left(x_{i}, 0\right)$ can be rewritten using a one-dimensional discrete operator $L_{x}^{h}$ applied to the unknown vector $\left\{e_{i}\right\}_{i=0}^{2 N_{0}}$ as

$$
\mathcal{L}^{h} e\left(x_{i}, 0\right)=L_{x}^{h} e_{i}:=\frac{\varepsilon^{2}}{h^{2}}\left[-e_{i-1}+2 e_{i}-e_{i+1}\right]+\left[\gamma_{i}+\frac{2 \varepsilon^{2}}{H^{2}}\right] e_{i} .
$$

On the other hand, $\mathcal{L}^{h} e\left(x_{i}, 0\right)=-\mathcal{L}^{h} u\left(x_{i}, 0\right)$, so the standard truncation error estimation (using $u\left(x_{i}, \pm H\right)=u\left(x_{i}, 0\right)$ ) yields

$$
\mathcal{L}^{h} e\left(x_{i}, 0\right)=\left[1-\gamma_{i}\right] u\left(x_{i}, 0\right)+\mathcal{O}\left(\frac{h^{2}}{\varepsilon^{2}}\right) .
$$

Here, by virtue of (3.2b), $\left[1-\gamma_{i}\right] u\left(x_{i}, 0\right)=\left[\frac{1}{3} \delta_{i N_{0}}\right] e^{-x_{i} / \varepsilon}=\frac{1}{3} e^{-1} \delta_{i N_{0}}$, where $\delta_{i N_{0}}$ denotes the Kronecker delta. So combining (3.6) and (3.7) yields

$$
L_{x}^{h} e_{i}=\frac{1}{3} e^{-1} \delta_{i N_{0}}+\mathcal{O}\left(\frac{h^{2}}{\varepsilon^{2}}\right) .
$$

As $L_{x}^{h}$ satisfies the discrete maximum principle [11, one gets the representation

$$
[\stackrel{\circ}{U}-u]\left(x_{i}, 0\right)=e_{i}=\frac{1}{3} e^{-1} h G_{i}^{h}+\mathcal{O}\left(\frac{h^{2}}{\varepsilon^{2}}\right) .
$$

Here $G_{i}^{h}$ is the discrete Green's function of the one-dimensional operator $L_{x}^{h}$ that satisfies $L_{x}^{h} G_{i}^{h}=h^{-1} \delta_{i N_{0}}$ for $i=1, \ldots, 2 N_{0}-1$, subject to $G_{0}^{h}=G_{2 N_{0}}^{h}=0$. To complete the proof of (3.5), it now suffices to show that $G_{N_{0}}^{h} \geq C_{3} \varepsilon^{-1}$ for some constant $C_{3}$; then one simply needs to choose sufficiently small constants $C_{0}$ and $C_{1}$ such that $\frac{1}{3} e^{-1} C_{3} \frac{h}{\varepsilon}+\mathcal{O}\left(\frac{h^{2}}{\varepsilon^{2}}\right) \geq 2 C_{0} \frac{h}{\varepsilon}$. (To be more precise, $C_{0}:=\frac{1}{12} e^{-1} C_{3}$, while, by virtue of $\frac{h}{\varepsilon}=N_{0}^{-1} \leq C_{1}$, we choose $C_{1}$ such that $\left|\mathcal{O}\left(\frac{h^{2}}{\varepsilon^{2}}\right)\right| \leq \frac{1}{3} e^{-1} C_{3} \frac{h}{\varepsilon}$.)

To bound $G_{N_{0}}^{h}$, note that $G_{i}^{h}=G_{2 N_{0}-i}^{h}$ and $G_{i}^{h}=G_{N_{0}}^{h}\left[w\left(x_{i} / \varepsilon\right)+\mathcal{O}\left(\frac{h^{2}}{\varepsilon^{2}}\right)\right]$ for $i \leq N_{0}$, where $w(x)$ solves the equation $-w^{\prime \prime}+\left(1+\frac{2 \varepsilon^{2}}{H^{2}}\right) w=0$ subject to $w(0)=0$ and $w(1)=1$. This implies that $\rho:=\frac{\varepsilon}{h}\left[G_{N_{0}}^{h}-G_{N_{0} \pm 1}^{h}\right] / G_{N_{0}}^{h} \leq C_{4}$ for some constant $C_{4}=C_{4}\left(C_{2}\right)$. (Roughly speaking, $\rho \approx w^{\prime}(1) \leq 1.32 \sqrt{1+2 C_{2}^{2}}$.) Now, $L_{x}^{h} G_{N_{0}}^{h}=h^{-1}$ can be rewritten as $G_{N_{0}}^{h}\left[2 \frac{\varepsilon}{h} \rho+\left(\frac{2}{3}+\frac{2 \varepsilon^{2}}{H^{2}}\right)\right]=h^{-1}$ or, equivalently, $G_{N_{0}}^{h}\left[2 \rho+O\left(\frac{h}{\varepsilon}\right)\right]=\varepsilon^{-1}$. As $\frac{h}{\varepsilon} \leq C_{1}$ and $\rho \geq C_{4}$, choosing $C_{1}$ sufficiently small we get $G_{N_{0}}^{h} \geq C_{3} \varepsilon^{-1}$ with $C_{3}:=\left(3 C_{4}\right)^{-1}$, and, hence, the desired bound (3.5). 

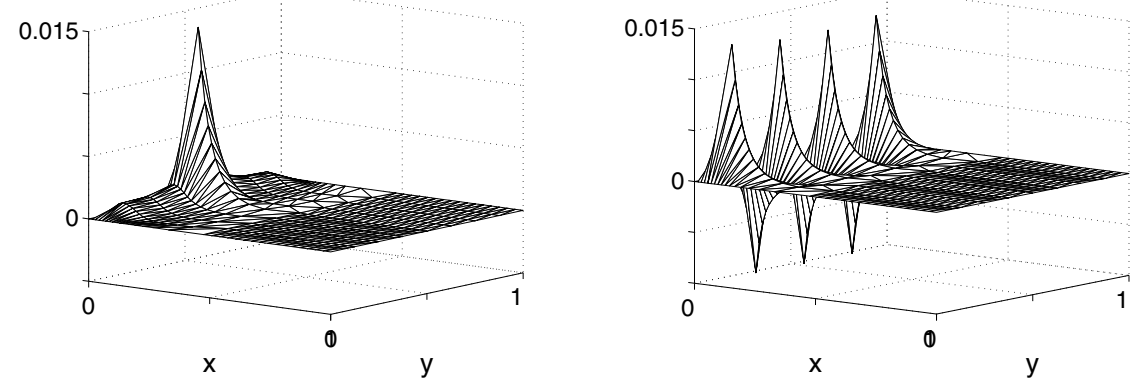

FiguRE 3. Pointwise computed-solution error on Triangulation B (left) and $\mathrm{C}$ (right), Bakhvalov tensor-product mesh, $\varepsilon=0.05, N=32$, $M=\frac{1}{4} N, \gamma=0.8$.

(ii) In view of (3.5), to establish (3.4), it suffices to show that

$$
\max _{\bar{\Omega}}\left|U-u^{I}\right| \geq \frac{1}{2} \max _{\Omega}\left|\stackrel{\circ}{\Omega}-u^{I}\right|=: \frac{1}{2} \stackrel{\circ}{ }
$$

where $u^{I}$ is the standard piecewise-linear interpolant of $u$.

Let $Z:=U-\stackrel{\circ}{U}$ in $\Omega$ and $Z_{\max }:=\sup _{\Omega}|Z|$. Note that $\mathcal{L}^{h} Z\left(x_{i}, 0\right)=0$ and $Z=U-u^{I}$ on $\partial \Omega$. As, by the discrete maximum principle, $|Z|$ attains its maximum

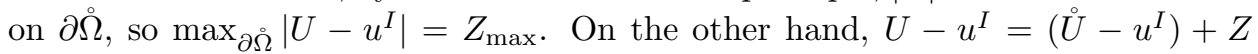
yields $\max _{\Omega}\left|U-u^{I}\right| \geq \stackrel{e}{e}-Z_{\max }$. As the maximum of the two values $\stackrel{\circ}{e}-Z_{\max }$ and $Z_{\max }$ exceeds their average, the desired relation (3.9) follows.

Remark 3.4. An inspection of the proof of Lemma 3.3 shows that if the assumption $\varepsilon \leq C_{2} H$ is replaced by a weaker $h \ll H$, one gets a version of (3.4) with $C_{0}$ replaced by $C_{0}^{\prime} / \sqrt{1+2 \frac{\varepsilon^{2}}{H^{2}}}$.

Remark 3.5. Lemma 3.3 applies to all triangulations of type B used in Tables 1-3, with $N_{0}^{-1}=O\left(N^{-1} \ln ^{p} \frac{N}{2}\right)$, where $p=0$ if the Bakhvalov mesh is used or the uniform mesh in the domain $\Omega=(0,2 \varepsilon) \times(0,1)$, and $p=1$ if the Shishkin mesh is used. Furthermore, this lemma still applies if one switches from Triangulation B to Triangulation $\mathrm{C}$.

Remark 3.6. For all triangulations considered in Section 2, one has $\varepsilon \ll M^{-1}$. So a version of part (i) of the proof of Lemma 3.3 (with negligible $\frac{\varepsilon^{2}}{H^{2}}$ ) can be applied to the global Triangulation B in $\Omega$ to show that $|[U-u](x, y)| \leq C\left(N^{-1} \ln ^{p} \frac{N}{2}\right)^{2}$ whenever $\left|y-\frac{1}{2}\right| \geq M^{-1}$, i.e., the deterioration in the accuracy occurs only near $y=\frac{1}{2}$; see Figure 3 (left). However, if one switches to Triangulation C, a similar analysis yields $[U-u]\left(x_{i}, \frac{j}{M}\right)=(-1)^{j} e_{i}$ with $e_{i}$ from (3.8) and a slightly different $G_{i}^{h}$ (now associated with the mesh $\left.\left\{x_{i}\right\}_{i=0}^{N}\right)$. Again, $\max e_{i}=O\left(N_{0}^{-1}\right)=O\left(N^{-1} \ln ^{p} \frac{N}{2}\right)$. So, as Figure 3 (right) demonstrates, the error of order (almost) 1 becomes spread throughout the boundary-layer region. Consequently, when the errors are computed in the $L_{2}$ norm, Triangulation B is superior to C (as shown in Table 4).

The computational convergence rates in Table 4 can be explained by a similar, but more detailed error analysis. To be more precise, with the notation $\mathcal{E}_{s}:=\left(N^{-1} \ln ^{p} \frac{N}{2}\right)^{s} e^{-C x / \varepsilon}$, it suffices to show that the error $\left|[U-u]\left(x, \frac{j}{M}\right)\right|$ roughly 
behaves like $\mathcal{E}_{2}$ for Triangulation $\mathrm{A}$, and like $\mathcal{E}_{1}$ for Triangulation $\mathrm{C}$, while for Triangulation B it behaves like $\mathcal{E}_{1}$ when $j=\frac{M}{2}$ and like $\mathcal{E}_{2}$ otherwise.

\subsection{Standard linear finite elements.}

Lemma 3.7 (Non-lumped-mass case). The statement of Lemma 3.3 remains valid for the computed solution $U$ obtained using standard linear finite elements.

Proof. We imitate the proof of Lemma 3.3 with a few changes described below.

(i) This part of the proof is devoted to establishing (3.5), only now $\stackrel{\circ}{U}\left(x_{i}, 0\right)$ satisfies (3.3), i.e., $\tilde{\mathcal{L}}^{h} \stackrel{\circ}{U}^{(3}\left(x_{i}, 0\right)=0$ for $i=1, \ldots, 2 N_{0}-1$. So a version of (3.6) for for $e:=\stackrel{\circ}{U}-u$ is obtained using the definition of $\tilde{\mathcal{L}}^{h}$ in (3.3) and the structure of the mesh (see Figure 2):

$$
\tilde{\mathcal{L}}^{h} e\left(x_{i}, 0\right)=\tilde{L}_{x}^{h} e_{i}:=\frac{\varepsilon^{2}}{h^{2}}\left(1-\frac{h^{2}}{12 \varepsilon^{2}}\right)\left[-e_{i-1}+2 e_{i}-e_{i+1}\right]+\left[\tilde{\gamma}_{i}+\frac{2 \varepsilon^{2}}{H^{2}}\right] e_{i} .
$$

Here $\tilde{\gamma}_{i}:=\gamma_{i}-\frac{4}{12}=\frac{2}{3}$ for $i \neq N_{0}$ and $\tilde{\gamma}_{N_{0}}:=\gamma_{N_{0}}-\frac{2}{12}=\frac{1}{2}$, while $\frac{h}{\varepsilon}=N_{0}^{-1}$ is assumed sufficiently small, so the one-dimensional discrete operator $\tilde{L}_{x}^{h}$ satisfies the discrete maximum principle and is handled similarly to $L_{x}^{h}$ of (3.6).

Next, by virtue of $\tilde{\mathcal{L}}^{h} e\left(x_{i}, 0\right)=-\tilde{\mathcal{L}}^{h} u\left(x_{i}, 0\right)$, a version of (3.7) is given by

$$
\tilde{\mathcal{L}}^{h} e\left(x_{i}, 0\right)=\left[\mathcal{L}^{h}-\tilde{\mathcal{L}}^{h}\right] u\left(x_{i}, 0\right)+\left[1-\gamma_{i}\right] u\left(x_{i}, 0\right)+\mathcal{O}\left(\frac{h^{2}}{\varepsilon^{2}}\right) .
$$

Note that the definition of $\tilde{\mathcal{L}}^{h}$ in (3.3) implies that

$$
\left[\mathcal{L}^{h}-\tilde{\mathcal{L}}^{h}\right] u\left(x_{i}, 0\right)=\frac{1}{12} \sum_{\left(x^{\prime}, y^{\prime}\right) \in \mathcal{S}_{i}}\left[u\left(x_{i}, 0\right)-u\left(x^{\prime}, y^{\prime}\right)\right],
$$

where $\mathcal{S}_{i}$ is the set of mesh nodes that have a common edge with $\left(x_{i}, 0\right)$. The righthand side here involves $u\left(x^{\prime}, \pm H\right)=u\left(x^{\prime}, 0\right)$, so, with the notation $u_{i}:=u\left(x_{i}, 0\right)$, a calculation using the structure of the mesh (see Figure 2) yields

$$
\left[\mathcal{L}^{h}-\tilde{\mathcal{L}}^{h}\right] u\left(x_{i}, 0\right)=\frac{1}{12}\left[-u_{i-1}+2 u_{i}-u_{i+1}\right]+\frac{1}{12} F_{i}=\mathcal{O}\left(\frac{h^{2}}{\varepsilon^{2}}\right)+\frac{1}{12} F_{i},
$$

where

$F_{i}:=\hat{\alpha}_{i}\left(u_{i}-u_{i+1}\right)+\check{\alpha}_{i}\left(u_{i}-u_{i-1}\right), \quad \hat{\alpha}_{i}:=\left\{\begin{array}{ll}2, & i<N_{0}, \\ 0, & i \geq N_{0},\end{array} \quad \check{\alpha}_{i}:= \begin{cases}0, & i \leq N_{0}, \\ 2, & i>N_{0} .\end{cases}\right.$

Next, define a decomposition $F_{i}=F_{i}^{\prime}+F_{i}^{\prime \prime}$ with

$$
F_{i}^{\prime}:=\left\{\begin{array}{cc}
-F_{2 N_{0}-i}, & i \leq N_{0} \\
F_{i}, & i \geq N_{0}
\end{array} \Rightarrow F_{i}^{\prime \prime}=\hat{\alpha}_{i}\left[\left(u_{i}-u_{i+1}\right)+\left(u_{2 N_{0}-i}-u_{2 N_{0}-i-1}\right)\right] .\right.
$$

Here, by virtue of $u_{j}=e^{-x_{j} / \varepsilon}$, one has $u_{j}-u_{j+1}=u_{j}\left(1-e^{-h / \varepsilon}\right)$ for any $j$, so one can easily check that $F_{i}^{\prime \prime} \geq 0$. Combining this with (3.10), (3.11) and (3.12) yields

$$
\tilde{L}_{x}^{h} e_{i}=\tilde{\mathcal{L}}^{h} e\left(x_{i}, 0\right) \geq \frac{1}{12} F_{i}^{\prime}+\left[1-\gamma_{i}\right] u\left(x_{i}, 0\right)+\mathcal{O}\left(\frac{h^{2}}{\varepsilon^{2}}\right) .
$$

To deal with $F_{i}^{\prime}$, let $\tilde{L}_{x}^{h} e_{i}^{\prime}=\frac{1}{12} F_{i}^{\prime}$ subject to $e_{0}^{\prime}=e_{2 N_{0}}^{\prime}=0$. The symmetry of this problem combined with the symmetry of $F_{i}^{\prime}$ immediately implies that $e_{N_{0}}^{\prime}=0$. Now $\tilde{L}_{x}^{h}\left[e_{i}-e_{i}^{\prime}\right] \geq\left[1-\gamma_{i}\right] u\left(x_{i}, 0\right)+\mathcal{O}\left(\frac{h^{2}}{\varepsilon^{2}}\right)$ yields a version of (3.5) for this equation $e_{N_{0}}-e_{N_{0}}^{\prime} \geq 2 C_{0} N_{0}^{-1}$ (with a different $C_{0}$ ). As $e_{N_{0}}^{\prime}=0$, the desired bound (3.5) follows.

(ii) This part of the proof is identical with part (ii) in the proof of Lemma 3.3 . To show that $|Z|$ attains its maximum on $\partial \Omega$, move the terms $Z_{i}^{ \pm}$, available from the boundary data, to the right-hand side in the discrete equation $\tilde{\mathcal{L}}^{h} Z=0$. The 
resulting nonhomogeneous one-dimensional discrete equation for the unknown vector $\left\{Z_{i}\right\}_{i=0}^{2 N_{0}}$ satisfies the discrete maximum principle, which can be used to deduce that the maximum of $|Z|$ indeed occurs on $\partial \Omega$.

\section{REFERENCES}

[1] V. B. Andreev, The logarithmic factor in the $L_{\infty}$-norm error estimate for linear finite elements is sharp, Semin. I. Vekua Inst. Appl. Math. Rep. (Dokl. Semin. Inst. Prikl. Mat. im. I. N. Vekua), 4 (1989), no. 3, 17-20 (in Russian).

[2] N. S. Bahvalov, On the optimization of the methods for solving boundary value problems in the presence of a boundary layer (Russian), Ž. Vyčisl. Mat. i Mat. Fiz. 9 (1969), 841-859. MR.0255066 (40 \#8273)

[3] H. Blum, Q. Lin, and R. Rannacher, Asymptotic error expansion and Richardson extrapolation for linear finite elements, Numer. Math. 49 (1986), no. 1, 11-37, DOI 10.1007/BF01389427. MR847015 (87m:65172)

[4] Long Chen, Pengtao Sun, and Jinchao Xu, Optimal anisotropic meshes for minimizing interpolation errors in $L^{p}$-norm, Math. Comp. 76 (2007), no. 257, 179-204, DOI 10.1090/S00255718-06-01896-5. MR2261017(2008e:65385)

[5] C. Clavero, J. L. Gracia, and E. O'Riordan, A parameter robust numerical method for a two dimensional reaction-diffusion problem, Math. Comp. 74 (2005), no. 252, 1743-1758, DOI 10.1090/S0025-5718-05-01762-X. MR2164094(2006e:65192)

[6] E. F. D'Azevedo, Optimal triangular mesh generation by coordinate transformation, SIAM J. Sci. Statist. Comput. 12 (1991), no. 4, 755-786, DOI 10.1137/0912040. MR.1102406 (92a:65041)

[7] E. F. D'Azevedo and R. B. Simpson, On optimal interpolation triangle incidences, SIAM J. Sci. Statist. Comput. 10 (1989), no. 6, 1063-1075, DOI 10.1137/0910064. MR 1025475 (91a:65019)

[8] Vít Dolejší and Jiří Felcman, Anisotropic mesh adaptation for numerical solution of boundary value problems, Numer. Methods Partial Differential Equations 20 (2004), no. 4, 576-608, DOI 10.1002/num.10104. MR2060780 (2005g:65182)

[9] Natalia Kopteva, Maximum norm error analysis of a 2D singularly perturbed semilinear reaction-diffusion problem, Math. Comp. 76 (2007), no. 258, 631-646 (electronic), DOI 10.1090/S0025-5718-06-01938-7. MR2291831(2008e:65327)

[10] Hans-Görg Roos, Martin Stynes, and Lutz Tobiska, Robust numerical methods for singularly perturbed differential equations, 2nd ed., Springer Series in Computational Mathematics, vol. 24, Springer-Verlag, Berlin, 2008. Convection-diffusion-reaction and flow problems. MR2454024(2009f:65002)

[11] A. A. Samarskiı̌, Teoriya raznostnykh skhem (Russian, with Russian summary), 3rd ed., "Nauka", Moscow, 1989. MR.1196231 (93g:65004)

[12] A. H. Schatz and L. B. Wahlbin, On the quasi-optimality in $L_{\infty}$ of the $\dot{H}^{1}$-projection into finite element spaces, Math. Comp. 38 (1982), no. 157, 1-22, DOI 10.2307/2007461. MR637283 (82m:65106)

[13] A. H. Schatz and L. B. Wahlbin, On the finite element method for singularly perturbed reaction-diffusion problems in two and one dimensions, Math. Comp. 40 (1983), no. 161, 47-89, DOI 10.2307/2007363. MR679434 (84c:65137)

[14] G. I. Shishkin, Grid approximation of singularly perturbed elliptic and parabolic equations, Ur. O. Ran, Ekaterinburg, 1992 (in Russian).

Department of Mathematics and Statistics, University of Limerick, Limerick, Ireland Current address: Department of Mathematics and Statistics, University of Strathclyde,

26 Richmond Street, Glasgow, G1 1XT, United Kingdom

E-mail address: natalia.kopteva@strath.ac.uk 